\title{
El estudio contemporáneo de la migración: realidades, enfoques y estrategias metodológicas emergentes. Introducción
}

\author{
Edith Y. Gutiérrez Vázquez*
}

M éxico es uno de los pocos países - probablemente el único- donde tienen lugar todo tipo de movimientos poblacionales, con escenarios migratorios complejos. Es un país de emigración e inmigración internacional, migración de retorno y de tránsito, y a la par experimenta un modelo mixto de migración interna en el que los flujos históricos ruralmetropolitano y urbano coexisten, aunque son de menor intensidad, con flujos significativos intermetropolitanos y de metrópolis a ciudades medias y entre estas mismas. La confluencia de todos estos movimientos en el territorio ha obedecido, entre otros factores, a los escenarios demográficos y económicos del país o son consecuencia de las políticas migratorias que tanto México como sus países vecinos han implementado (Canales, 2015; Giorguli, Guerrero \& Masferrer, 2016).

En términos de migración internacional, a partir de la década de los noventa del siglo pasado, México ha experimentado la intensificación de diversos flujos migratorios. El flujo de mexicanos a Estados Unidos se acentuó en esa década hasta convertirse en el movimiento internacional del Sur al Norte global más cuantioso del mundo. En 2015, los mexicanos residentes en Estados Unidos sumaban 12 millones y representaban aproximadamente un tercio de todos los latinos que vivían en el país vecino (American Community Survey, 2010), conformando así una minoría étnica con fuertes lazos con sus comunidades de origen e importantes montos de remesas, que impulsan una agenda de investigación sobre incorporación de migrantes. Esta realidad ha movido la agenda de investigación de visiones unidireccionales (desde el origen o desde el destino) de los procesos a visiones transnacionales (origen-destino y destino-origen), y paradigmas de asimilación y

Profesora-investigadora del Departamento de Estudios Regionales-Ineser, Centro Universitario de Ciencias Económico Administrativas, Universidad de Guadalajara, edith.gutierrez@cucea.udg.mx 
aculturación en procesos de integración que consideran un conjunto de indicadores de bienestar más allá de los indicadores socioeconómicos, como el estado de salud física y mental o la participación social de los migrantes.

El reforzamiento de las políticas migratorias de deportación y cierre de fronteras, aunado al aliento de un clima antiinmigrante y a la crisis económica de 2008, que golpeó severamente los nichos laborales de los migrantes mexicanos en Estados Unidos, trajo consigo un incremento significativo de la migración de retorno, lo cual llevó la tasa de migración neta a niveles cercanos a cero, los más bajos registrados recientemente (Parrado y Gutiérrez, 2016). Este flujo transformó la agenda de investigación de retorno, México no solo no había recibido tantos connacionales en edades laborales (más de un millón de personas), sino que el discurso prevaleciente hasta entonces era el del paradigma clásico - no resueltode la migración y el desarrollo, en el que los migrantes o se convierten en motor de desarrollo local o por lo menos generan sus propias oportunidades laborales. Sin embargo, el contexto de origen del retorno, permeado primordialmente por la involuntariedad del movimiento, transformó las necesidades y los recursos de los retornados, quienes ahora se suman a las filas de la población que busca oportunidades laborales en el país. La migración internacional ha venido dejando de ser la válvula de escape del mercado laboral mexicano.

A la par de la desaceleración de la migración de mexicanos a Estados Unidos, principalmente del flujo de no autorizados, se ha venido observando un incremento en el flujo de migración centroamericana al país vecino del norte. Las estimaciones más recientes de Passel y Cohn (2017) señalan que los migrantes de Centroamérica y Asia, junto con los de otras nacionalidades, y no los mexicanos, son los que ahora predominan en el contingente de población que ingresa a Estados Unidos sin autorización. Este incremento significa claramente un ascenso del flujo de personas que transita por México hacia Estados Unidos, fenómeno que, a pesar de estar presente desde los años ochenta por los conflictos civiles de los países que conforman la región central del continente, ha sido poco estudiado en comparación con otros fenómenos migratorios nacionales debido, entre otros factores, a la carencia de fuentes y la dificultad para medirlo dada su clandestinidad. El mayor flujo de migración de tránsito mueve la agenda de investigación sobre migración a enfrentar actores complejos; no solo son actores que buscan transitar en el anonimato sino también son flotantes, puesto que están en constante movimiento, y en contextos de la creciente vulnerabilidad y violencia, dado el clima prevaleciente en México, y de incertidumbre en cuanto al cruce, debido al endurecimiento del control de la frontera estadounidense.

En estos mismos periodos, la redistribución territorial de la población dio un vuelco importante: mientras que a mediados de los cincuenta poco más de la mitad 
de la población vivía en zonas rurales, en años recientes el $78 \%$ de los habitantes residen en urbes o metrópolis (INEGI, 2010). La transición rural-urbana se ha dado en un contexto de diversificación de los flujos de destinos más a menos urbanizados y entre ellos, es decir, el flujo rural-metropolitano e intermetropolitano ha descendido para dar cabida a un flujo metropolitanociudades medias (Sobrino, 2010). Esta realidad ha suscitado diversas teorías que buscan explicar cómo la migración lleva $-\mathrm{y}$ hacia dónde- el binomio concentración-dispersión de la población. En este sentido, las agendas de investigación se han movido hacia la integración multidisciplinaria de perspectivas y técnicas metodológicas para dar espacio a la espacialización —análisis espacial-de fenómenos sociales.

Los artículos de este número y de los dos próximos avanzan en la agenda de investigación contemporánea sobre migración al abordar preguntas investigativas en temas como nuevos actores migratorios, cambios en las tendencias y dinámicas migratorias, desafíos de integración, innovaciones metodológicas, interrelaciones de la migración y otros eventos del curso de vida que son consecuencia de la dinámica migratoria y los retos que esta representa para las políticas públicas y de migración. En particular, en este número se publican tres artículos que abonan a los avances metodológicos en tres temáticas migratorias: migración de tránsito, migración interna y el vínculo entre migración y fecundidad. El primero contribuye a la medición del tiempo de tránsito y espera al cruce de centroamericanos que atraviesan México rumbo a Estados Unidos. Este trabajo es el primero en cuantificar esta característica temporal de la migración de tránsito de manera sistemática con la fuente de información de mayor cobertura, aunque de representatividad limitada, disponible actualmente sobre la migración centroamericana que llega a Estados Unidos. Estas estimaciones tienen implicaciones importantes en materia de política pública, en cuanto a la provisión de servicios humanitarios básicos a esta población de estancia temporal y a la garantía de sus derechos humanos. El segundo artículo aporta el diseño de una metodología híbrida para combinar el carácter espacial de la migración -localización de destinos y orígenes y la distancia entre estos- y los conocimientos de los actores migratorios sobre esta espacialidad, a la par de las características de los destinos potenciales para la toma de decisiones sobre el destino final de la migración. Su contribución metodológica permite entender mejor el comportamiento migratorio individual y mejorar las predicciones de los flujos y la redistribución territorial de la población, que son importantes para la planeación de programas y políticas. El tercer artículo, al presentar una extensa síntesis de la investigación sobre el vínculo entre migración y fecundidad, demuestra cómo la complejidad del fenómeno migratorio ha impulsado la 
innovación metodológica en áreas como selectividad, determinantes asociados a fenómenos, y fuentes y análisis de corte longitudinal. La migración se establece como un fenómeno demográfico que determina, media e interfiere con otros fenómenos demográficos y procesos sociales. Estos distintos roles de la variable migratoria, y en específico en su vínculo con la fecundidad, alientan la creación y combinación de métodos de la disciplina demográfica con otros de la estadística, como la integración del método de hijos propios y las técnicas de regresión o adaptación de modelos longitudinales para el entendimiento de las interrelaciones entre fenómenos, así como el uso de metodologías cuantitativas, cualitativas y mixtas, y la creación ingeniosa de contrafactuales para la medición adecuada de la asociación de la fecundidad con la migración independiente de características que determinan la migración per se, e inclusive a la fecundidad.

Además de las contribuciones metodológicas, cada uno de los tres artículos aporta a un tema amplio de la migración. Rosalba Jasso y César Barboza, en su artículo sobre migración de tránsito por México, muestran cómo las definiciones y estrategias de políticas migratorias en este país y en el vecino han modificado los tiempos de traslado de la migración centroamericana en el territorio nacional, a la par de haber redireccionado el flujo por rutas que incrementan la vulnerabilidad de los migrantes, estableciendo así nuevos lugares de espera para el cruce e incrementando la duración de las estancias en ellos. El entendimiento y la medición de este componente temporal es de suma relevancia porque la migración centroamericana hacia Estados Unidos ha venido al alza (Passel y Cohn, 2017), lo cual implica que los montos de migrantes que atraviesan el país también se ha incrementado. A la par, en su tránsito, esta población enfrenta los retos de satisfacer sus necesidades en contextos de clandestinidad y de la violencia prevaleciente en el país, retos que a su vez generan demandas sociales, de garantías de los derechos humanos e incluso económicas, puesto que algunos de estos migrantes no cuentan con suficiente capital monetario para cubrir los costos del cruce o lo han agotado en el trayecto. La modificación de los tiempos de tránsito y espera al cruce, en especial su incremento, tienen pues consecuencias sociales, políticas y económicas directas y en diversas áreas geográficas del país, las cuales se intensifican dependiendo de si el territorio forma parte de la ruta de tránsito o es un punto de cruce. Los autores utilizan los años de 2009 a 2014 de la Encuesta sobre Migración en la Frontera Sur (Emif Sur) en su apartado sobre migrantes devueltos de Estados Unidos. En esta población analizan el tiempo que les tomó transitar por México, su punto de cruce a Estados Unidos y las ciudades fronterizas en las que permanecieron y el tiempo de permanencia previo al cruce.

Jasso y Barboza encuentran que el endurecimiento de las políticas migratorias de México y Estados Unidos ha forzado a la población migrante de 
Centroamérica a demorarse más en transitar y cruzar al país vecino. Además, con la transformación de las rutas en México, debida al reforzamiento del control migratorio en el territorio más allá de la frontera sur y las barreras crecientes impuestas en la frontera con Estados Unidos, principalmente en puntos tradicionales de cruce como Tijuana-San Isidro, se han redefinido las ciudades del norte de México en las que los centroamericanos aguardan para cruzar, del noroeste al noreste del país. La redefinición regional de estos puntos de cruce tiene implicaciones de índole académica, socioeconómica y gubernamental; surgen preguntas en contextos no explorados antes, y que requieren acciones focalizadas regionalmente; por ejemplo ¿cuáles son, y cuáles debieran ser, las prácticas y medidas que los gobiernos locales, estatales y federal deben ejercer para controlar el flujo a la par de garantizar la seguridad y bienestar de estas personas?, ¿cómo enfrentar las necesidades laborales temporales de esta población flotante en los puntos de cruce?, ¿cómo coadyuvar a la labor y los esfuerzos de organizaciones no gubernamentales y civiles en la provisión de servicios humanitarios básicos (salud, vivienda y alimentación) y la previsión de la victimización de la población migrante en tránsito? Fuentes como la Emif Sur nos pueden dar la pauta para entender los elementos, actores y procesos implicados en la migración de tránsito del sur al norte de México.

Por su parte, Estela Rivero y Marcos Valdivia exploran preguntas metodológicas fundamentales en la migración, centrándose en el fenómeno migratorio interno. ¿Cómo deciden los migrantes el destino al que se dirigen del conjunto de destinos potenciales para emigrar? ¿El conjunto de destinos es ilimitado o está restringido por el conocimiento que los migrantes tienen de estos, las condiciones de los mismos, y de ser así, por cuáles condiciones? Las metodologías actuales limitan las posibilidades de tomar en cuenta las distintas opciones y la información que los migrantes en potencia tienen para tomar la decisión sobre el destino. A este fenómeno los autores lo denominan heterogeneidad de los tomadores de decisiones en un contexto dinámico, y se aproximan en su operacionalización simulando contextos en los que los migrantes potenciales poseen información variable sobre estos destinos en términos de su contexto urbano, las amenidades y los servicios que ofertan, y la distancia de estos respecto al lugar de origen de los migrantes. Complejizan la heterogeneidad simulando escenarios donde varían el número de destinos de que los migrantes poseen información, contribuyendo así a una paradoja importante de la teoría de las migraciones, ¿en el tiempo, al descender la intensidad migratoria intermetropolitana y diversificarse a otros destinos, la distribución territorial de los migrantes se equilibra, es decir, observamos un equilibrio espacial de migrantes? Para responder esta pregunta utilizan información de los censos de población de 2000 y 2010 para alimentar un método híbrido 
que combina elementos de sistemas de información geográfica — para el trazo de la red potencial de destinos- y la simulación de toma de decisiones de agentes migratorios artificiales (con características demográficas y ubicación reales).

Sus resultados apuntan a la diversificación de los flujos y un declive de la concentración de la migración en los flujos intermetropolitanos. Sin embargo, en contraposición a la hipótesis de equilibrio espacial de migrantes, encuentran que los migrantes en potencia deciden entre un grupo de destinos limitado, principalmente delimitado por la proximidad de destinos según su jerarquía urbana, es decir, por la distancia de estos respecto de sus lugares de origen y las amenidades y los servicios que ofertan. Este resultado es de suma relevancia no solo para las teorías sobre migración sino para los estudios regionales, ya que posiciona los procesos de regionalización - creación y consolidación de zonas donde se aglomeran y concentran economías y servicios de toda índole-como determinantes fundamentales de la distribución territorial de la población por medio de la influencia que ejercen sobre las decisiones de migración de la población.

Finalmente, Ricardo Régules sistematiza los cambios en los enfoques, las estrategias metodológicas, las fuentes de información y los hallazgos de la investigación entre la migración interna e internacional y la fecundidad. En esta revisión Régules muestra cómo, pese a ser un tema de estudio desde los años treinta del siglo pasado, a partir de la década de los setenta la búsqueda del entendimiento de las interrelaciones de fenómenos demográficos, que también forman parte de eventos fundamentales del curso de vida individual, ha impulsado la innovación en técnicas, diseño de nuevas fuentes y diversificación de enfoques. El autor divide su revisión entre los estudios de migración interna e internacional, aunque los enfoques teórico-explicativos presentados como hipótesis de su investigación coinciden para ambos fenómenos. Analiza la socialización, selectividad, separación, adaptación y aculturación para cada fenómeno migratorio en sus componentes explicativos, mediciones y fuentes de información. De su revisión identificamos que los enfoques de la literatura de migración y fecundidad podrían agruparse entre explicaciones asociadas a las condiciones previas a la migración (como la socialización y selectividad) y las posteriores a ella, incluyendo aquellas que son consecuencia directa del hecho de emigrar por sí mismo, como la separación, adaptación y aculturación. En el texto también identificamos que la evidencia actual apunta a que la migración incide en la fecundidad primordialmente por medio de la alteración de algunas de sus variables intermedias asociadas a su ritmo, tempo (para una revisión de estas variables se puede consultar el artículo seminal de Davis y Blake, 1956), más que a la limitación directa en el número total de hijos, quantum. La migración altera el calendario de la fecundidad de los individuos al postergar y modificar el espaciamiento de sus nacimientos en forma distinta del 
calendario observado en los no migrantes, ya sea de manera directa, por la separación del núcleo conyugal, o indirecta, por la interrelación de la migración con procesos sociales como la incorporación de normas sociales y culturales o el enfrentamiento de barreras económicas y las sociedades de destino, y con otros eventos del curso de vida, como la entrada de las mujeres al mercado laboral para el apoyo del hogar expuesto a la migración.

Para avanzar en estas agendas se han diseñado creativas estrategias metodológicas ante la escasez de información longitudinal binacional. Considerando la condición migratoria de la población, se han combinado técnicas demográficas, como el método de hijos propios para la estimación de fecundidad, con técnicas estadísticas de modelaje, para poner a prueba los enfoques y determinantes asociados a la migración y la fecundidad. Asimismo se han adaptado técnicas longitudinales, también llamadas de historia de eventos, para profundizar en el entendimiento de la interrelación de la migración, la fecundidad y otros eventos del curso de vida tomando en cuenta la temporalidad y el ritmo, además de otros determinantes del fenómeno de estudio. Entre estos avances también se encuentran el desarrollo de fuentes de información longitudinal y las mejoras en la medición y en los instrumentos de captación sobre la historia de vida de las personas.

Un hallazgo adicional de la sistematización que se debe enfatizar es la importancia de las características tanto del origen como del destino de la migración para el entendimiento de la reducción de la fecundidad y de la magnitud de la misma. Entre estas características, los estudios apuntan al nivel de urbanización de ambas locaciones, origen y destino, como determinante indispensable en la adaptación de la fecundidad. Dado el carácter regional de la migración, tanto de los orígenes como de los destinos (véase Durand y Massey, 2003), cabe preguntarse si también esta característica de la migración ha contribuido a generar o reproducir comportamientos diferenciados territorialmente de la fecundidad, y sus subsecuentes contribuciones a patrones demográficos regionales. Esta es una agenda de investigación compartida entre las disciplinas de los estudios regionales y de la demografía.

Las transformaciones de la migración en México impulsan nuevas agendas de investigación que plantean retos para las formas clásicas de análisis y entendimiento del fenómeno. Se necesita analizar temas tradicionales y emergentes de la migración desde perspectivas bidireccionales y de circuitos (origen-destino y destino-origen), incluir y diseñar distintas medidas del bienestar, las necesidades y el comportamiento de los migrantes que nos permitan entender las causas y consecuencias del movimiento estudiándolas desde perspectivas más amplias que priorizan la integración de estos agentes sociales y la reducción de su vulnerabili- 
dad, y que consideran los aspectos objetivos y subjetivos de esta integración. En tal sentido, los tres artículos reseñados de este número señalan pendientes en cuanto a información y métodos para el avance de la agenda investigativa. Jasso y Barboza puntualizan que la Emif Sur no es completamente representativa de la población de tránsito porque no están representados ni los migrantes que cruzan y logran establecerse en Estados Unidos ni los que regresan voluntariamente a sus lugares de origen. Se necesita información sobre estas subpoblaciones para tener la visión completa de la población de tránsito por México. Rivero y Valdivia señalan la necesidad de incorporar la causalidad simultánea de la migración y el desarrollo de los lugares de destino. Los migrantes no solo son atraídos por amenidades y servicios sino que también se convierten en un polo de atracción para el desarrollo de la oferta de dichas amenidades y servicios. Se necesita desarrollar metodologías que permitan integrar la endogeneidad de estos dos fenómenos, migración y desarrollo (económico en este caso) para llegar a conclusiones contundentes sobre las tendencias de la distribución de migrantes y la selección de destinos. Por su parte, Régules enfatiza la necesidad de medir aspectos subjetivos de la migración, en especial aspectos sociales y psicoemocionales que pueden incidir en los comportamientos reproductivos y la fecundidad de las personas migrantes, la combinación de información sobre las experiencias previas, durante y posteriores a la migración, y la combinación de metodologías cuantitativas y cualitativas para dar cuenta con mayor certeza de la complejidad que representa el estudio de interrelaciones entre fenómenos cambiantes en el curso de vida.

\section{Referencias}

Canales, A. (2015). E pur si mouve. Elementos para una teoría de las migraciones en el capitalismo global. México: Universidad de Guadalajara, Miguel Ángel Porrúa.

Davis, K. \& Blake, J. (1956). Social structure and fertility: an analytic framework. Economic and Cultural Change, 4(2), 211-235.

Durand, J. \& Massey, D. (2003). Clandestinos. Migración México-Estados Unidos en los albores del siglo XXI. México: Miguel Ángel Porrúa.

Giorguli Saucedo, S., García Guerrero, V. \& Masferrer, C. (2016). A migration system in the making: demographic dynamics and migration policies in North America and the Northern Triangle of Central-America. México: El Colegio de México. 
Parrado, E. A. \& Gutiérrez, E. (2016). The changing nature of return migration to Mexico, 1990-2010: implications for labor market incorporation and development. Sociology ofDevelopment, 2(2), 93-118.

Passel, J. \& Cohn, D. (2017). As Mexican share declined, U.S. unauthorized immigrant population fell in 2015 below recession level. Fact tank. News in the numbers. Pew Research Center. Recuperado de http://pewrsr.ch/2qavmFK (consultado el 26 de septiembre de 2017).

Sobrino, J. (2010). Migración interna en México durante el siglo XX. México: Consejo Nacional de Población. 